\title{
An exact solution method for Fredholm integro-differential equations
}

\author{
K. D. Tsilikaa, PhD, Assistant Professor, orcid.org/0000-0002-9213-3120, ktsilika@uth.gr \\ a Laboratory of Operations Research, Department of Economics, University of Thessaly, Volos, 38221, Greece
}

Introduction: Linear boundary value problems for Fredholm ordinary integro-differential equations are seldom considered with integral boundary conditions in the literature. In our case, integro-differential equations are subject to multipoint or nonlocal integral boundary conditions. It should be noted that finding exact solutions even for multipoint problems or problems with nonlocal integral boundary conditions with a differential equation is a difficult task. Purpose: Finding the uniqueness and existence criterion of solutions for Fredholm ordinary integro-differential equations with multipoint or nonlocal integral boundary conditions and obtaining exact solutions in closed form of such problems. Results: Within the class of abstract operator equations, for the special case of Fredholm integro-differential equations with multipoint or nonlocal integral boundary conditions, a criterion for the existence and uniqueness of an exact solution is proved and the analytical representation of the solution is given. A direct method analytically solving such problems is proposed, in which all calculations are reproducible in any program of symbolic calculations. If the user sets the input parameters and the initial conditions of the problem, the computer codes check the conditions of existence and uniqueness and of solution generate the analytical solution. The stages of the solution method are illustrated by two examples. The article uses computer algebra system Mathematica to demonstrate the results.

Keywords - boundary value problems, ordinary differential equations, Fredholm integro-differential equations, multipoint boundary conditions, nonlocal integral boundary conditions, correct operators, exact solutions.

For citation: Tsilika K. D. An exact solution method for Fredholm integro-differential equations. Informatsionno-upravliaiushchie sistemy [Information and Control Systems], 2019, no. 4, pp. 2-8. doi:10.31799/1684-8853-2019-4-2-8

\section{Introduction}

Consider the boundary value problem of the type

$$
\begin{gathered}
B u=A u-\mathbf{g F}(A u)=f, D(B)=\{u \in D(A): \\
\Phi(u)=\mathbf{N} \Psi(u)\}, u \in X, f \in Y,
\end{gathered}
$$

where $A$ is an ordinary differential operator; $\mathbf{F}(A u)=\operatorname{col}\left(F_{1}(A u), \ldots, F_{n}(A u)\right)$ is a functional vector representing the integral part of the integro-differential equation; $\mathbf{g}=\left(g_{1}, \ldots, g_{n}\right)$ is a vector; $\mathbf{N}$ - a constant $m \times l$ matrix; $\Phi=\operatorname{col}\left(\Phi_{1}, \ldots, \Phi_{m}\right) ; \Psi=\operatorname{col}\left(\Psi_{1}, \ldots, \Psi_{l}\right)$ are functional vectors with $\Psi(u)$ standing for the multipoint or integral part of the boundary conditions.

Note that problem (1) describes the particular case of the multipoint boundary value problem with integro-differential equation (IDE) (see [1]) given below

$$
\begin{gathered}
u^{(m)}(x)+\sum_{i=1}^{m} p_{i}(x) u^{(m-i)}(x)+ \\
+\sum_{i=0}^{m} \int_{a}^{b} K_{i}(x, y) u^{(m-i)}(y) \mathrm{d} y=f(x) ; \\
\sum_{j=0}^{v+1} \sum_{i=1}^{m} a_{k i j} u^{(i-1)}\left(x_{j}\right)+\left(u, \varphi_{k}\right)=0, \\
k=1, \ldots, s,\left(a=x_{0}<x_{1}<\ldots<x_{v+1}=b\right),
\end{gathered}
$$

where $\left(u, \varphi_{k}\right)$ is an integral part of the boundary conditions. IDEs like (2) are investigated also in [2]. In [1, 2] the existence theorem for (2) has been proved. This problem is reduced to problem (1) if $s=m, v=0, K_{i}(x, y)=g(x) h(y) p_{i}(y), i=0,1, \ldots, m$, $p_{0}(x)=1$ and $\left\|a_{k i 0}\right\|_{k, i=1}^{m}=\mathbf{I}_{m}$.

Indeed, then we can take

$$
\begin{gathered}
A u=u^{(m)}(x)+\sum_{i=1}^{m} p_{i}(x) u^{(m-i)}(x) ; \\
\Phi_{i}(u)=u^{(i-1)}\left(x_{0}\right), i=1, \ldots, m, \\
\Psi_{i(u)}=\left\{\begin{array}{l}
u^{(i-1)}(x), i=1, \ldots, m \\
\left(u, \varphi_{i-m}\right), i=m+1, \ldots, 2 m
\end{array}\right\},
\end{gathered}
$$

$\mathbf{F}(A u)=\int_{a}^{b} h(y)\left[u^{(m)}(y)+\sum_{i=1}^{m} p_{i}(y) u^{(m-i)}(y)\right] \mathrm{d} y$ and $N=-\left\|a_{k i 1}, \delta_{k i}\right\|_{k, i=1}^{m}, \delta_{k i}$ is a Kronecker delta function.

In this paper, we study the solvability of problem (1) and analytically construct its solution. The present work lies in the field of boundary value problem for linear integral equations [3, 4]. In this setting, problems are often too complex to be solved analytically and therefore numerical solutions are proposed $[5,6]$. Analytical solutions of a class of boundary value problems, involving the governing differential equation or slight generalizations of the one considered in (1), but with different and/or simpler differential operators, have been proposed in [7-10]. Existence theorems for nonlocal multipoint boundary value problem and IDEs via analytical methods are given in [11-13]. Boundary value problems $B$ : $X \rightarrow X$ of the type of (1) for the specific case of $l=n$ 
have been studied by Vassiliev, Parasidis, Providas in $[14,15]$, using the extension method. The extension method is a generalization of the direct method, which is presented in [4]. Here, problem (1) is investigated and solved for the case $l \neq n, X \neq Y$ mainly by functional transformations and by employing the properties of linear operators. The results of this method coincide with the results of $[14,15]$ for $l=n$.

The novelties of the present paper are the following. First we address the local boundary value problem of the type of (1) without restrictions to the dimensions $l, n, m$; second, we assume multipoint or nonlocal integral boundary conditions, which allows us to consider a very large class of problems for the equation (1). The ultimate result is the exact solution of problem (1).

We describe now the setting of our approach. Let $X, Y$ be complex Banach spaces and $X^{*}$ the adjoint space of $X$, i. e. the set of all complex-valued linear and bounded functionals on $X$. We denote by $f(x)$ the value of $f$ on $x . D(A)$ and $R(A)$ are, respectively, the domain and the range of the operator $A$. The following definitions are extremely important in the analysis of the next section.

Definition 1. An operator $A_{2}$ is said to be an extension of an operator $A_{1}$, or $A_{1}$ is said to be a restriction of $A_{2}$, in symbol $A_{1} \subset A_{2}$, if $D\left(A_{2}\right) \supset D\left(A_{1}\right)$ and $A_{1} x=A_{2} x$, for all $x \in D\left(A_{1}\right)$.

Definition 2. An operator $A: X \rightarrow Y$ is called closed if for every sequence $x_{n} \in D(A)$ converging to $x_{0}$ with $A x_{n} \rightarrow f_{0}$ follows that $x_{0} \in D(A)$ and $A x_{0}=f_{0}$.

Definition 3. A closed operator $A$ is called maximal if $R(A)=Y$ and $\operatorname{ker} A \neq 0$.

Definition 4. An operator $\hat{A}: X \rightarrow Y$ is called correct if $R(\hat{A})=Y$ and the inverse $\hat{A}^{-1}$ exists and is continuous on $Y$.

Definition 5. An operator $\hat{A}$ is called a correct restriction of the maximal operator $A$ if it is a correct operator and $\hat{A} \subset A$.

Basic notations. If $\Psi_{i} \in X^{*}, i=1, \ldots, l$, then we consider the functional vectors $\Psi=\operatorname{col}\left(\Psi_{1}, \ldots, \Psi_{l}\right)$ and $\Psi(x)=\operatorname{col}\left(\Psi_{1}(x), \ldots, \Psi_{l}(x)\right)$. Let $g=\left(g_{1}, \ldots g_{n}\right)$ be a vector of $X^{n}$. Hereafter we will denote by $\Psi(g)$ the $l \times n$ matrix whose $i, j$-th entry $\Psi_{i}\left(g_{j}\right)$ is the value of functional $\Psi_{i}$ on element $g_{j}$. Note that $\Psi(\mathrm{gC})=\Psi(\mathrm{g}) \mathbf{C}$, where $\mathbf{C}$ is a $n \times k$ constant matrix. We will also denote by $\mathbf{I}_{n}$ the identity $n \times n$. By $\mathbf{0}$ we will denote the zero column vector. We will denote by $\operatorname{ker} A$ the $\operatorname{ker}-$ nel of an operator $A$.

\section{Exact solution method for Fredholm IDEs}

Suppose that $A: X \rightarrow X$ is an ordinary $m$-order differential operator

$$
A u(x)=a_{0} u^{(m)}(x)+a_{1} u^{(m-1)}(x)+\ldots+a_{m} u(x), a_{i} \in R,(3)
$$

$X=C[a, b]$ or $X=L_{p}(a, b), p \geq 1, \mathbf{z}=\left(z_{1}, z_{2}, \ldots, z_{m}\right)$ a basis of $\operatorname{ker} A$ and $\hat{A}$ be a correct restriction of $A$ defined by

$$
\hat{A} \subset A, D(\hat{A})=\{u \in D(A): \Phi(u)=\mathbf{0}\},
$$

where the components of the functional vector $\Phi=\operatorname{col}\left(\Phi_{1}, \ldots, \Phi_{m}\right)$, belong to $\left[X_{A}^{m}\right]^{*}$ and are biorthogonal to $z_{1}, \ldots, z_{m}$. Everywhere below we denote by $X_{A}^{m}=C^{m}[a, b]$ if $X=C[a, b]$, or $X_{A}^{m}=W_{p}^{m}(a, b)$ if $X=L_{p}(a, b)$ and by $\left[X_{A}^{m}\right]^{*}$ we denote the adjoint to $X_{A}^{m}$ space, i. e. the space of all linear bounded functionals defined on $X_{A}^{m}$. It is a well-known fact that the operator $\hat{A}: C[a, b] \rightarrow C[a, b]$ defined by

$$
\begin{gathered}
\hat{A} u(x)=a_{0} u^{(m)}(x)+a_{1} u^{(m-1)}(x)+\ldots+a_{m} u(x)=f, \\
a_{i} \in R, x \in[a, b], \\
D(\hat{A})= \\
=\left\{u(x) \in C^{m}[a, b]: u(a)=u^{\prime}(a)=\ldots=u^{(m-1)}(a)=0\right\}, \\
a_{0} \neq 0
\end{gathered}
$$

is a correct restriction of $A$ and the unique solution of (5) for $a_{0}=1, a_{1}=\ldots=a_{m}=0, a=0$ is

$$
\begin{gathered}
u(x)=\hat{A}^{-1} f(x)=\frac{1}{(m-1) !} \int_{a}^{x}(x-t)^{m-1} f(t) \mathrm{d} t \\
f(x) \in \mathbf{C}[0, b]
\end{gathered}
$$

Before we address the issue of finding the exact analytic solutions of the Fredholm IDE, we will mention briefly some properties of the linear operators of these equations.

Lemma 1. Let $A$ be a differential operator defined by (3), $\mathbf{z}=\left(z_{1}, \ldots, z_{m}\right)$ a basis of $\operatorname{ker} A, \mathbf{N}$ a $m \times l$ matrix with constant elements, the components of a vector $\Psi=\operatorname{col}\left(\Psi_{1}, \ldots, \Psi_{l}\right)$ be functionals on $X_{A}^{m}$, the matrix $\mathbf{V}=\mathbf{I}_{l}-\Psi(\mathbf{z}) \mathbf{N}$ and the operator $C: X_{A}^{m} \rightarrow X_{A}^{m}$ be defined by

$$
C u=u-\mathbf{z N} \Psi(u) \text {. }
$$

Then:

(i) The operator $C$ is invertible if and only if $\operatorname{det} \mathbf{V} \neq 0$;

(ii) the inverse operator $C^{-1} v=v+\mathbf{z N V}^{-1} \Psi(v)$.

Proof: Let $\operatorname{det} \mathbf{V} \neq 0$ and $u \in \operatorname{ker} C$. Then $u-\mathbf{z N} \Psi(u)=\mathbf{0} \quad$ and $\quad \Psi(u-\mathbf{z N} \Psi(u))=\mathbf{0}, \quad \Psi(u)-$ $-\Psi(\mathbf{z}) \mathbf{N} \Psi(u)=\mathbf{0}, \quad\left[\mathbf{I}_{l}-\Psi(\mathbf{z}) \mathbf{N}\right] \Psi(u)=\mathbf{0}$. From the last equation since $\operatorname{det} \mathbf{V} \neq \mathbf{0}$ follows that $\Psi(u)=\mathbf{0}$. Substituting this value into $u-\mathbf{z N} \Psi(u)=0$ we get $u=0$. Consequently, $\operatorname{ker} C=\{0\}$ and $C$ is invertible. 
Conversely, let $\operatorname{det} \mathbf{V}=0$. Then there exists a vector $\mathbf{c} \neq \mathbf{0}$ such that $\mathbf{V c}=\mathbf{0}$.

Consider the element $u_{0}=\mathbf{z N c}$. Note that $u_{0} \neq 0$, otherwise the linear independence of the components of the vector $\mathbf{z}$ implies $\mathbf{N c}=\mathbf{0}$ and then $\mathbf{V c}=\left[\mathbf{I}_{l}-\Psi(\mathbf{z}) \mathbf{N}\right] \mathbf{c}=\mathbf{c}-\Psi(\mathbf{z}) \mathbf{N c}=\mathbf{c}-\mathbf{0}=\mathbf{c}=\mathbf{0} . \quad$ This contradicts the hypothesis that $\mathbf{c} \neq \mathbf{0}$. So, $u_{0} \neq \mathbf{0}$. We will show that $u_{0} \in \operatorname{ker} C$. Observe that $C u_{0}=u_{0}-$ $-\mathbf{z N} \Psi\left(u_{0}\right)=\mathbf{z N c}-\mathbf{z N} \Psi(\mathbf{z}) \mathbf{N c}=\mathbf{z N}\left[\mathbf{I}_{l}-\Psi(\mathbf{z}) \mathbf{N}\right] \mathbf{c}=$ $=\mathbf{z N V c}=\mathbf{z N 0}=\mathbf{0}$. Thus $u_{0} \in \operatorname{ker} C$ and $\operatorname{ker} C \neq\{0\}$. Hence $C$ is not invertible. So $C$ is invertible if and only if $\operatorname{det} \mathbf{V} \neq \mathbf{0}$. Let $C u=v$, i. e. $u-\mathbf{z N} \Psi(u)=v$. Then $\Psi(u)-\Psi(\mathbf{z}) \mathbf{N} \Psi(u)=\Psi(v)$, or $\left[\mathbf{I}_{l}-\Psi(\mathbf{z}) \mathbf{N}\right] \Psi(u)=\Psi(v)$, which implies $\Psi(u)=\mathbf{V}^{-1} \Psi(v)$. From the last equation and $u-\mathbf{z N} \Psi(u)=v$ follows that $u=v+\mathbf{z N} \mathbf{V}^{-1} \Psi(v)$, which yields $u=C^{-1} v$. The lemma is proved.

The next theorem is analogous to theorem which has been proved in [8] when $F_{1}, \ldots, F_{n}$ are linearly independent. This theorem is already proved for the linearly independent set $g_{1}, \ldots, g_{n}$, though using a different method. In addition, it is proved here that the criterion for correctness of the operator $B$ in [8] coincides with the criterion for injectivity of $B$.

Theorem 1. Let $X, Y$ be complex Banach spaces, $\hat{A}: X \rightarrow Y$ be a correct linear operator, the functional vector $\mathbf{F}=\operatorname{col}\left(F_{1}, \ldots, F_{n}\right) \in\left[Y^{*}\right]^{n}$, a vector $\mathbf{g}=\left(g_{1}\right.$, $\left.\ldots, g_{n}\right) \in Y^{n}$ and $g_{1}, \ldots, g_{n}$ is a linearly independent set. Then:

(i) The operator $B$ defined by

$$
B u=\hat{A} u-\mathbf{g} \mathbf{F}(\hat{A} u)=f, \quad D(B)=D(\hat{A}), f \in Y
$$

is injective if and only if

$$
\operatorname{det} \mathbf{W}=\operatorname{det}\left[\mathbf{I}_{n}-\mathbf{F}(\mathbf{g})\right] \neq \mathbf{0}
$$

(ii) If $B$ is injective, then $B$ is correct and the unique solution of (7) is given by

$$
u=B^{-1} f=\hat{A}^{-1} f+\left(\hat{A}^{-1} \mathbf{g}\right)\left[\mathbf{I}_{n}-\mathbf{F}(\mathbf{g})\right]^{-1} \mathbf{F}(f), f \in Y .
$$

Proof: (i) Let $\operatorname{det} \mathbf{W}=0$ and $u=\operatorname{ker} B$, i. e. $B u=\hat{A} u-\mathbf{g F}(\hat{A} u)=0$. Since $g_{i} \in R(\hat{A})=Y, \quad i=1$, ..., $n$, by using (7) we have $\mathbf{F}(\hat{A} u)-\mathbf{F}(\mathbf{g}) \mathbf{F}(\hat{A} u)=\mathbf{0}$ and $\left[\mathbf{I}_{n}-\mathbf{F}(\mathbf{g})\right] \mathbf{F}(\hat{A} u)=\mathbf{0}$. Taking into account that $\operatorname{det} \mathbf{W} \neq 0$, from the last equation we get $\mathbf{F}(\hat{A} u)=\mathbf{0}$. Then $B u=\hat{A} u=0$ and $u=0$. The last follows from $\operatorname{ker} \hat{A}=\{0\}$. So $\operatorname{ker} B=\{0\}$ and $B$ is an injective operator.

Conversely. Let $\operatorname{det} \mathbf{W} \neq 0$. Then there exists a vector $\mathbf{c}=\operatorname{col}\left(c_{1}, \ldots, c_{n}\right) \neq \mathbf{0}$ such that $\mathbf{W c}=\mathbf{0}$. Note that $\mathbf{g c} \neq \mathbf{0}$ because $g_{1}, \ldots, g_{n}$ is a linearly independent set. Then $u_{0}=\hat{A}^{-1} \mathbf{g c} \neq 0$ and $B u_{0}=\hat{A} u_{0}-\mathbf{g F}\left(\hat{A} u_{0}\right)=\mathbf{g c}-\mathbf{g F}(\mathbf{g}) \mathbf{c}=\mathbf{g}\left[\mathbf{I}_{n}-\mathbf{F}(\mathbf{g})\right] \mathbf{c}=$ $=\mathbf{g W c}=\mathbf{g} \mathbf{0}=\mathbf{0}$.
Hence $u_{0}=\hat{A}^{-1}$ gc $\in \operatorname{ker} B \neq\{0\}$ and $B$ is not injective. So statement (i) holds.

(ii) Let $\operatorname{det} \mathbf{W} \neq 0$. Then from (7) we get

$$
\begin{gathered}
\mathbf{F}(\hat{A} u)-\mathbf{F}(\mathbf{g}) \mathbf{F}(\hat{A} u)=\mathbf{F}(f), \\
{\left[\mathbf{I}_{n}-\mathbf{F}(\mathbf{g})\right] \mathbf{F}(\hat{A} u)=\mathbf{F}(f),} \\
\mathbf{F}(\hat{A} u)=\mathbf{W}^{-1} \mathbf{F}(f) \text { for all } f \in Y ; \\
u-\hat{A}^{-1} \mathbf{g F}(\hat{A} u)=\hat{A}^{-1}(f) .
\end{gathered}
$$

Substituting (10) into (11), we obtain the unique solution (9) of (7) for all $f \in Y$. Then $R(\hat{A})=Y$. The boundedness of $B^{-1}$ on $Y$ results from the boundedness of the operator $\hat{A}^{-1}$ and the components of vector $\mathbf{F}$. Hence, $B$ is correct.

Now we generalize this theorem for the case of perturbed boundary conditions and $\operatorname{dim} \operatorname{ker} A=m$. The next theorem is a generalization of the Theorem 1 of [14] for the case $l \neq n$ and $X \neq Y$.

Theorem 2. Let $X, Y$ be complex Banach spaces, $A: X \rightarrow Y$ be an operator from (3) with finite dimensional kernel $\mathbf{z}=\left(z_{1}, \ldots, z_{m}\right)$ which is a basis of $\operatorname{ker} A$, the operator $\hat{A}$ defined by (4) and the components of the functional vectors $\Phi=\operatorname{col}\left(\Phi_{1}, \ldots, \Phi_{m}\right)$, $\Psi=\operatorname{col}\left(\Psi_{1}, \ldots, \Psi_{l}\right)$, and $\mathbf{F}=\operatorname{col}\left(F_{1}, \ldots, F_{n}\right)$ belong to $\left[X_{A}^{m}\right]^{*}$ and $Y^{*}$ respectively. Suppose also that $\Phi_{1}$, ..., $\Phi_{m}$ are biorthogonal to $z_{1}, \ldots, z_{m}$ and the components of vector $\mathbf{g}=\left(g_{1}, \ldots, g_{n}\right) \in Y^{n}$ are linearly independent and $\mathbf{N}$ is a $m \times l$ matrix.

Then: (i) The operator $B$ defined by

$$
\begin{gathered}
B u=A u-\mathbf{g F}(A u)=f, f \in Y ; \\
D(B)=\{u \in D(A): \Phi(u)=\mathbf{N} \Psi(u)\}
\end{gathered}
$$

is injective if and only if

$$
\begin{aligned}
& \operatorname{det} \mathbf{V}=\operatorname{det}\left[\mathbf{I}_{l}-\mathbf{\Psi}(\mathbf{z}) \mathbf{N}\right] \neq 0 \\
& \operatorname{det} \mathbf{W}=\operatorname{det}\left[\mathbf{I}_{n}-\mathbf{F}(\mathbf{g})\right] \neq 0
\end{aligned}
$$

(ii) If $B$ is injective, then $B$ is correct and for all $f \in Y$ the unique solution of (12) is given by

$$
\begin{aligned}
u=B^{-1} f & =\hat{A}^{-1} f+\left[\hat{A}^{-1} \mathbf{g}+\mathbf{z N} \mathbf{V}^{-1} \Psi\left(\hat{A}^{-1} \mathbf{g}\right)\right] \times \\
\times & W^{-1} \mathbf{F}(f)+\mathbf{z} \mathbf{N} \mathbf{V}^{-1} \Psi\left(\hat{A}^{-1} f\right)
\end{aligned}
$$

Proof: (i) The problem (12) can be rewritten as

$$
\begin{gathered}
B u=A(u-\mathbf{z N} \Psi(u))-\mathbf{g F}(A[u-\mathbf{z N} \Psi(u)])=f, f \in Y, \\
D(B)=\{u \in D(A): \Phi(u-\mathbf{z N} \Psi(u))=0\} .
\end{gathered}
$$

Then $u-\mathbf{z N} \Psi(u) \in \mathrm{D}(\hat{A}), \quad B u=\hat{A}(u-\mathbf{z N} \Psi(u))-$ $-\mathbf{g} F(\hat{A}[u-\mathbf{z N} \Psi(u)])=f$ for every $u \in D(B)$. We introduce the substitution $v=u-\mathbf{z N} \Psi(u)$ or since 
Lemma 1, $v=C u$, where we take $C: X \rightarrow X, D(C)=$ $=D(B)$. It is evident that $C$ is a linear operator and $R(C) \subseteq D(\hat{A})$. From (16) it follows that problem (12), by the substitution $v=C u$, is reduced to the problem

$$
B u=B_{1} v=\hat{A} v-\mathbf{g F}(\hat{A} v)=f, v \in D\left(B_{1}\right)=D(\hat{A}) .
$$

Since $v=C u$, from (17) we get $B u=B_{1} C u$ for all $u \in D(B)$. Then $B$ is injective if and only if $B_{1}$ and $C$ are also injective. Note that the operator $C$, by Lemma 1 , is injective if and only if $\operatorname{det} \mathbf{V}=\operatorname{det}\left[\mathbf{I}_{l}-\mathbf{\Psi}(\mathbf{z}) \mathbf{N}\right] \neq \mathbf{0}$. The operator $B_{1}$, by Theorem 1 , where instead of $B, u$ we have $B_{1}, v$ respectively, is injective if and only if (8) holds. So the operator $B$ is injective if and only if (13), (14) hold true.

(ii) The unique solution of (17) by (9) for all $f \in Y$ is given by

$$
v=\hat{A}^{-1} f+\left(\hat{A}^{-1} \mathbf{g}\right) \mathbf{W}^{-1} \mathbf{F}(f) .
$$

Then

$$
\Psi(v)=\Psi\left(\hat{A}^{-1} f\right)+\Psi\left(\hat{A}^{-1} \mathbf{g}\right) \mathbf{W}^{-1} \mathbf{F}(f)
$$

and since $\operatorname{det} \mathbf{V} \neq 0, v=u-\mathbf{z N} \Psi(u)$, by Lemma 1 , we get

$$
\begin{gathered}
u=C^{-1} v=v+\mathbf{z N} \mathbf{V}^{-1} \Psi(v)= \\
=\hat{A}^{-1} f+\left(\hat{A}^{-1} \mathbf{g}\right) \mathbf{W}^{-1} \mathbf{F}(f)+ \\
+\mathbf{z N V} \mathbf{V}^{-1}\left[\Psi\left(\hat{A}^{-1} f\right)+\Psi\left(\hat{A}^{-1} \mathbf{g}\right) \mathbf{W}^{-1} \mathbf{F}(f)\right]
\end{gathered}
$$

The last equation easily implies the unique solution of (12) defined by (15). The operator $B_{1}$, by Theorem 1 , is correct. Then $R\left(B_{1}\right)=Y$, which since $B u=B_{1} v$ gives $R(B)=Y$. Since the operator $\hat{A}^{-1}$ and the functionals $F_{1}, \ldots, F_{n}, \Psi_{1}, \ldots, \Psi_{l}$ are bounded, the boundedness of $B^{-1}$ follows from (15). Hence, the operator $B$ is correct. This completes the proof of Theorem 2.

Now, we implement the solution method of Theorem 2 on two numerical examples, the first involving a linear second order differential operator and the other a linear forth order differential operator $A$.

Example 1. Consider the multipoint problem for loaded differential equation on $C[0,1]$

$$
\begin{gathered}
u^{\prime \prime}-\left(3 t^{3}-2 t\right)\left[u^{\prime}(1)-u(1)+u(0)\right]= \\
=-6 t^{3}+4 t+4, \\
u(0)=2 u(1)+u(0.5)-2 u^{\prime}(1), \\
u^{\prime}(0)=-u(1)-2 u(0.5)+3 u^{\prime}(1) .
\end{gathered}
$$

All the assumptions of Theorem 2 hold and the unique solution of (21) is

$$
u(t)=2 t^{2}-5 t+3
$$

Proof: If we compare (21) with (12), it is natural to take $X=Y=C[0,1]$,

$$
\begin{aligned}
& B u=u^{\prime \prime}-\left(3 t^{3}-2 t\right)\left[u^{\prime}(1)-u(1)+u(0)\right]= \\
& =u^{\prime \prime}-\left(3 t^{3}-2 t\right) \int_{0}^{1} x u^{\prime \prime}(x) \mathrm{d} x, \\
& D(B)= \\
& =\left\{u(x) \in D(A):\left(\begin{array}{c}
u(0) \\
u^{\prime}(0)
\end{array}\right)=\left(\begin{array}{ccc}
2 & 1 & -2 \\
-1 & -2 & 3
\end{array}\right)\left(\begin{array}{c}
u(1) \\
u(0.5) \\
u^{\prime}(1)
\end{array}\right)\right\} \text {, } \\
& A u=u^{\prime \prime}(t), D(A)=\left\{u \in C^{2}[0,1]\right\}, \\
& X_{A}^{m}=C^{2}[0,1], X=C[0,1], m=2, l=3, n=1 \text {, } \\
& \mathbf{z}=\left(z_{1}, z_{2}\right)=(1, t), \hat{A} u=A u, \\
& D(\hat{A})=\left\{u \in D(A): u(0)=u^{\prime}(0)=0\right\} \text {. }
\end{aligned}
$$

The operator $\hat{A}$ is correct and, since (6), its solution is $\hat{A}^{-1} f(t)=\int_{0}^{t}(t-x) f(x) \mathrm{d} x$. Further comparing (21), (23) with (12), we take $g(t)=3 t^{3}-2 t$, $f(t)=-6 t^{3}+4 t+4, \quad \mathbf{N}=\left(\begin{array}{ccc}2 & 1 & -2 \\ -1 & -2 & 3\end{array}\right), \quad \mathbf{F}(A u)=$
$=\int_{0}^{1} x u^{\prime \prime}(x) \mathrm{d} x$.

The solution method of Theorem 2 for problem (21) is implemented in computer algebra system Mathematica (v. 11.3) [16]. The Mathematica notebook containing the solution method is available upon request.

Even though Mathematica has a dedicated function to symbolically solve an integro-differential equation (solves also Fredholm integral equations), DSolveValue (new feature in Mathematica v. 11, see https://www.wolfram.com/language/11/symbolic-and-numeric-calculus/solve-an-integro-differential-equation.html?product = language), in this example fails to give an output. Trying to take the analytic solution with NDSolveValue instead, the procedure gave a bunch of errors. Built-in function NDSolve failed also.

In the Mathematica codes given below, we provide an overview of the symbolic computations we employed for the illustrative Example 1. The computer codes give to the reader a computerized approach along with the analytical one. All of the commands have quite similar analogues in the case of any order integro-differential equation. It is then possible to create a script for solving this type of integro-differential equations. Here, only the main ideas and procedures for the solution are given and the basic knowledge of how to use Mathematica is assumed.

In Mathematica's environment, we begin by writing the functions that represent the structural elements of the operator equation (21). Then we de- 
fine the functional vectors of the boundary conditions. Functions and functional vectors are written in a way to take various elements as arguments. In Example 1, $t$ takes values from the interval $[0,1]$. The criterion for injectivity of $B$ that is tested and verified is the only requirement to apply Theorem 2 and formulate the unique solution as in (15).

Mathematica code

in $[*]:=(*$ definitions $*)$

in [*]: $=(*$ Give the order $m$ of the differential operator $A$ or the number of conditions or the dimension $m$ of the functional vector $\Phi^{*}$ )

$\operatorname{in}[*]:=m=2$;

in $[*]:=(*$ Give the dimension $l$ of the functional vector $\Psi *)$

$\operatorname{in}[*]:=l=3$;

in $[*]:=(*$ Give the dimension $n$ of the functional vector $\mathrm{F} *$ )

$\operatorname{in}[*]:=n=1$;

in $[*]:=(*$ Define the structural elements of the operator equation $\left.A u-g F(A u)=f^{*}\right)$

$\operatorname{in}[*]:=(* F$ is the integral part of the IDE*)

in $[*]=F\left[\right.$ function $\left._{-}\right]:=\left\{\int_{0}^{1} x *\right.$ function $\left.\mathrm{d} x\right\}$

$\operatorname{in}[*]:=f\left[t_{-}\right]:=\left\{-6 * t^{3}+4 * t+4\right\}$

$\operatorname{in}[*]:=g\left[t_{-}\right]:=\left\{3 * t^{3}-2 * t\right\}$

in $[*]:=(*$ Give the values of the variables in the boundary conditions*)

in $[*]:=t i=\{1,0.5,1\}$;

in $\left[{ }^{*}\right]:=(*$ Give the $m \times l N$ matrix "nmatrix" such that $\left.\Phi(u)=N \Psi(u)^{*}\right)$

$\operatorname{in}[*]:=$ nmatrix $=\{\{2,1,-2\},\{-1,-2,3\}\} ;$

in [*]: $=(*$ Give the functional vector $\Psi$ such that $\left.\Phi(u)=N \Psi(u)^{*}\right)$

$\operatorname{in}\left[{ }^{*}\right]:=\Psi[$ function_]: $=\{$ function $/ . t \rightarrow t i[[1]]$,

function $/ . t \rightarrow t i[[2]], D[$ function, $t] / . t \rightarrow t i[[3]]\}$

$\operatorname{in}[*]:=(*$ The solution method $*)$

$\operatorname{in}[*]:=W:=$ IdentityMatrix $[n]-F[g[x]]$

in [*]: $=V:=$ IdentityMatrix $[l]-\Psi[\mathrm{z}]$. nmatrix

in [*]: = z: = Table $\left[t^{i} / i !,\{i, 0, m-1\}\right]$;

in $[*]=$ inverse $A\left[\right.$ function $\left._{-}\right]:=$

$=\frac{1}{(m-1) !} \int_{0}^{t}(t-x)^{m-1} *$ function $\mathrm{d} x$

in $[*]:=(*$ Verify the assumptions of Theorem $2 *)$ in [*]: $=(*$ Testing necessary and sufficient conditions for operator $B u=A u-g F(A u)$ to be injective*) $\operatorname{in}[*]:=\operatorname{Det}[W]$

out $[*]=\frac{16}{15}$

$\operatorname{in}[*]:=\operatorname{Det}[V]$

out $[*]:=1.5$

in $[*]:=(*$ Testing the existence and uniqueness criterion*)

$\operatorname{in}[*]:=\operatorname{If}[\operatorname{Det}[W] \neq 0 \quad \& \& \operatorname{Det}[V] \neq 0$, “The IDE has a unique solution", "The solution is not unique"] out [*]: = The IDE has a unique solution

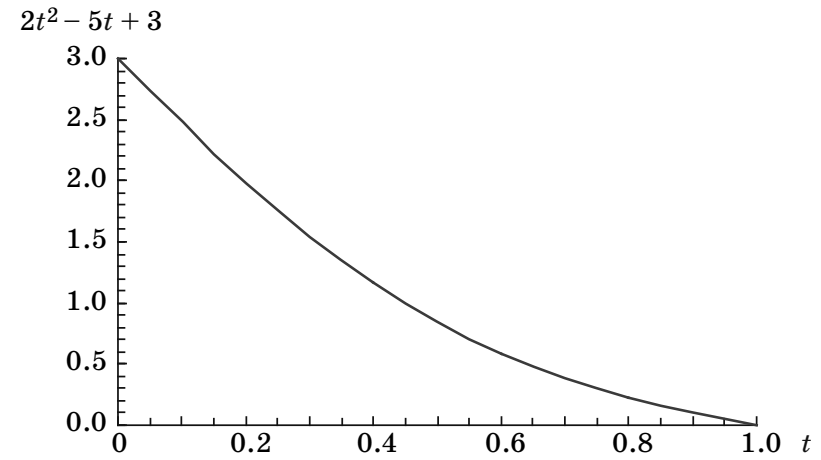

Values for $u(t)$ over the domain $[0,1]$

in $[*]:=(*$ Here is the unique solution by the exact solution method*)

in [*]: = solution: $=$ Simplify[inverse $A[f[x]]+($ inverse $A[g[x]]+$ z.n matrix.Inverse $[V]$. $\Psi[\operatorname{inverse} A[g[x]]])$.Inverse[W]. $F[f[x]]+$ z.nmatrix. Inverse $[V] . \Psi[$ inverse $A[f[x]]]]$

in [*]: = Print["The exact solution of the IDE is" Flatten[solution]]

\{The exact solution of the IDE is $\left.\left(3 .-5 . t+2 t^{2}\right)\right\}$

The graphical representation of the analytical solution to this problem is shown in Figure:

in $\left[{ }^{*}\right]=\operatorname{Plot}\left[3-5 t+2 t^{2},\{t, 0,1\}\right.$, AxesLabel $\rightarrow\{t$, $\left.\left.3-5 t+2 t^{2}\right\}\right]$.

Example 2. Consider the multipoint problem for differential equation on $C[0,1]$

$$
\begin{gathered}
u^{(4)}(t)-\left(t^{2}-4 t+1\right) \times \\
\times \int_{0}^{1} x u^{(4)}(x) \mathrm{d} x=-12 t^{2}+48 t+12, \\
u(0)=3 u(1), u^{\prime}(0)=-5 u^{\prime}(1)+u^{\prime \prime}(1), \\
u^{\prime \prime}(0)=-\frac{1}{5} u^{\prime \prime}(1), u^{\prime \prime \prime}(0)=24 u^{\prime \prime}(1)-10 u^{\prime \prime \prime}(1) .
\end{gathered}
$$

The unique solution of (24) is

$$
u(t)=t^{4}-t^{2}
$$

We solve problem (24) using the previous Mathematica script, by changing only the input parameters $m, l, n, \mathbf{F}, f(t), g(t), \mathbf{N}, \Psi(u)$. The program checks if all the assumptions of Theorem 2 hold and the unique solution is determined explicitly in finitely many steps of computation, following (15).

Mathematica code

in $[*]=(*$ definitions $*)$

$\operatorname{in}[*]=(*$ Give the order $m$ of the differential operator $A$ / number of conditions or the dimension $m$ of the functional vector $\Phi^{*}$ )

$\operatorname{in}[*]=m=4$;

$\operatorname{in}[*]=(*$ Give the dimension $l$ of the functional vector $\Psi *$ )

$\operatorname{in}\left[{ }^{*}\right]=l=4$ 
$\operatorname{in}[*]=(*$ Give the dimension $n$ of the functional vector $\mathrm{F}^{*}$ )

$\operatorname{in}[*]=n=1$;

in $[*]=(*$ Define the structural elements of the operator equation $A u=g F(A u)=f *)$

$\operatorname{in}[*]=(* \mathrm{~F}$ is the integral part of the IDE*)

in $[*]=F[$ function $]:=\left\{\int_{0}^{1} x *\right.$ functiond $\left.x\right\}$

$\operatorname{in}[*]=f[t]:=\left\{-12 * t^{2}+48 * t+12\right\}$

$\operatorname{in}[*]=g\left[t_{-}\right]:=\left\{t^{2}-4 * t+1\right\}$

$\operatorname{in}[*]=(*$ Give the values of the variables in the boundary conditions*)

$\operatorname{in}[*]=t i=\{1,1,1,1\}$;

in $[*]=(*$ Give the $m \times l N$ matrix "nmatrix" such that $\left.\Phi(u)=N \Psi(u)^{*}\right)$

in $[*]=$ nmatrix $=\{\{3,0,0,0\},\{0,-5,1,0\},\{0,0$,

$-1 / 5,0\},\{0,0,24,-10\}\}$;

in $[*]=(*$ Give the functional vector $\Psi$ such that $\left.\Phi(u)=N \Psi(u)^{*}\right)$

$\operatorname{in}[*]=\Psi[$ function_ $]:=\{$ function $/ . t \rightarrow t i[[1]] ;$ $\mathrm{D}[$ function, $t] / . t \rightarrow t i[[2]], D[$ function, $\{t, 2\}] / . t \rightarrow$

$\rightarrow t i[[3]], D[$ function, $\{t, 3\}] / . t \rightarrow t i[[4]]\}$

$\operatorname{in}[*]=(*$ The solution method $*)$

$\operatorname{in}[*]=W:=$ IdentityMatrix $[n]-F[g[x]]$

$\operatorname{in}[*]=V:=$ IdentityMatrix $[l]-\Psi[z]$. nmatrix

in $\left.{ }^{*}\right]=\mathrm{z}:=$ Table $\left[t^{i} / i !,\{i, 0, m-1\}\right]$;

in $[*]=$ inverse $A[$ function_]: =

$=\frac{1}{(m-1) !} \int_{0}^{t}(t-x)^{m-1} *$ function $\mathrm{d} x$

in $[*]=(*$ Verify the assumptions of Theorem $2 *)$

$\operatorname{in}[*]=(*$ Testing necessary and sufficient condi-

tions for operator $B u=A u-g F(A u)$ to be injective*) $\operatorname{in}[*]=\operatorname{Det}[W]$

\section{References}

1. Parkhimovich I. V. Multipoint boundary value problems for linear integro-differential equations in the class of smooth functions. Differentsial'nye uravneniya [Differential Equations], 1972, vol. 8, no 3, pp. 549-552 (In Russian).

2. Tamarkin J. D. The notion of the Green's function in the theory of integro-differential equations. Transactions of the American Mathematical Society, 1927, vol. 29 , no. 4 pp. $755-800$.

3. Polyanin A. D., Zhurov Alexei I. Exact solutions to some classes of nonlinear integral, integro-functional and integro-differential equation. Doklady Mathematics, 2008, vol. 77, no. 2, pp. 315-319. doi:10.1134/ S1064562408020403

4. Wazwaz A. M. Linear and Nonlinear Integral Equations. Methods and Applications. Springer, Beijing, 2011. $657 \mathrm{p}$.

5. Dzhumabaev D. S. On one approach to solve the linear boundary value problems with Fredholm integro-differential equations. Journal of Computational and

$$
\begin{aligned}
& \text { out }[*]=\frac{19}{12} \\
& \operatorname{in}[*]=\operatorname{Det}[V] \\
& \text { out }[*]=\frac{648}{5}
\end{aligned}
$$

$\operatorname{in}[*]:=(*$ Testing the existence and uniqueness criterion*)

$\operatorname{in}[*]:=\operatorname{If}[\operatorname{Det}[W] \neq 0 \& \& \operatorname{Det}[V] \neq 0$, “The IDE has a unique solution", "The solution is not unique"] out $[*]:=$ The IDE has a unique solution

in [*]: $=\left({ }^{*}\right.$ Here is the unique solution by the exact solution method*)

in $[*]=$ solution: $=$ Simplify $[$ inverse $A[f[x]]+($ inverse $A[g[x]]+$ z.n matrix.Inverse $[V]$. $\Psi[\operatorname{inverse} A[g[x]]])$.Inverse $[W] . F[f[x]]+$ z.nmatrix. Inverse $[V] . \Psi[$ inverse $A[f[x]]]]$

$\operatorname{in}\left[{ }^{*}\right]=$ Print[“The exact solution of the IDE is" Flatten[solution]]

$\left\{\right.$ The exact solution of the IDE is $\left.t^{2}\left(-1+t^{2}\right)\right\}$

in $[*]=$ Expand[solution[[1]]]

out $\left[{ }^{*}\right]=-t^{2}+t^{4}$

\section{Conclusion}

In the paper, an analytical method for giving exact solutions for Fredholm integro-differential equations is presented. This analytical method is constructive in the sense that it allows to study the unique solvability of the problem and analytically construct its solution. A couple of numerical examples were solved by this method in Mathematica software. Reproducibility of the proof is possible through the Mathematica script.

Applied Mathematics, 2016, vol. 294, pp. 342-357. doi: https://doi.org/10.1016/j.cam.2015.08.023

6. Dhamacharoen A., Chompuvised K. An efficient method for solving integro-differential boundary value problems. AIP Conference Proceedings 1982, 2018, pp. 020015-1-020015-11.

7. Oinarov R. O., Parasidi I. N. Correct extensions of operators with finite defect in Banach spases. Izvestiya Akademii Nauk Kazakhskoi SSR, 1988, no. 5, pp. 42-46 (In Russian).

8. Parasidis I. N., Tsekrekos P. C. Some quadratic correct extensions of minimal operators in Banach space. Operators and Matrices, 2010, vol. 4, no. 2, pp. 225-243. doi:dx.doi.org/10.7153/oam-04-11

9. Parasidis I. N., Tsekrekos P. C. Correct and self-adjoint problems for quadratic operators. Eurasian Mathematical Journal, 2010, vol. 1, no. 2, pp. 122135.

10. Parasidis I. N., Providas E. Extension Operator Method for the Exact Solution of Integro-Differential Equations. In: Pardalos P., Rassias T. (eds). Contributions in Mathematics and Engineering: In Honor of 
Constantin Caratheodory. Springer, 2016. P. 473496. doi:10.1007/978-3-319-31317-7_23

11. Abdullaev A. R., Skachkova E. A. On one class of multipoint boundary value problems for a second-order linear functional-differential equation. Journal of Mathematical Sciences, 2018, vol. 230, no. 5, pp. 647-650.

12. Benchohra M., Ntouyas S. K. Existence results on the semiinfinite interval for first and second order integro-differential equations in Banach spaces with nonlocal conditions. Acta Universitatis Palackianae Olomucensis, Facultas Rerum Naturalium, Mathematica, 2002, vol. 41, pp. 13-19.

13. Ionkin N. I. The solution of a certain boundary value problem of the theory of heat conduction with a nonclassical boundary condition. Differentsial'nye uravneniya [Differential Equations], 1977, vol. 13, no. 2, pp. 294-304 (In Russian).
14. Vassiliev N. N., Parasidis I. N., Providas E. Exact solution method for Fredholm integro-differential equations with multipoint and integral boundary conditions. Part 1. Extension method. Informatsionno-upravliaiushchie sistemy [Information and Control Systems], 2018, no. 6, pp. 14-23. doi:10.31799/ 1684-8853-2018-6-14-23

15. Vassiliev N. N., Parasidis I. N., Providas E. Exact solution method for Fredholm integro-differential equations with multipoint and integral boundary conditions. Part 2. Decomposition-extension method for squared operators. Informatsionno-upravliaiushchie sistemy [Information and Control Systems], 2019, no. 2, pp. 2-9. doi:10.31799/1684-8853-2019-2-2-9

16. Wolfram Research, Inc. Mathematica, Version 11.3, Champaign, Illinois, 2018.

УДК 338.984

doi:10.31799/1684-8853-2019-4-2-8

Метод точного решения для интегро-дифференциальных уравнений Фредгольма

К. Д. Тсилика ${ }^{\mathrm{a}}, \mathrm{PhD}$, доцент, orcid.org/0000-0002-9213-3120, ktsilika@uth.gr

ауниверситет Фессалии, 38221, Волос, Греция

Введение: линейные краевые задачи с интегральными граничными условиями для дифференциальных и интегро-дифференциальных уравнений Фредгольма редко рассматриваются в литературе. Представляется важным исследование интегро-дифференциальных уравнений с многоточечными или нелокальными интегральными граничными условиями. Даже для дифференциальных уравнений получение точных решений с подобными граничными условиями является непростой задачей. Цель: поиск критерия существования и единственности решений для обыкновенных интегро-дифференциальных уравнений Фредгольма с сепарабельным ядром и многоточечными или нелокальными интегральными граничными условиями; разработка математической методики, ведущей к точным аналитическим решениям заданного уравнения. Результаты: для одного класса абстрактных операторных уравнений, частным случаем которых являются интегро-дифференциальные уравнения Фредгольма с многоточечными или нелокальными интегральными граничными условиями, получены критерий существования и единственности точного решения и его аналитическое представление; предложен прямой метод, символически решающий подобные задачи, в котором все вычисления воспроизводимы в любой программе символьных вычислений. Если пользователь устанавливает входные параметры и начальные условия задачи, выделенные компьютерные коды проверяют условия существования и единствености и генерируют аналитическое решение задачи. Этапы решения проиллюстрированы двумя примерами. В статье использовано программное обеспечение CAS Mathematica.

Ключевые слова - краевые задачи, обыкновенные дифференциальные уравнения, интегро-дифференциальные уравнения Фредгольма с сепарабельным ядром, многоточечные граничные условия, нелокальные интегральные граничные условия, корректные операторы, точные решения.

Для цитирования: Tsilika K. D. An exact solution method for Fredholm integro-differential equations. Информационно-управляющие системы, 2019, № 4, с. 2-8. doi:10.31799/1684-8853-2019-4-2-8

For citation: Tsilika K. D. An exact solution method for Fredholm integro-differential equations. Informatsionno-upravliaiushchie sistemy [Information and Control Systems], 2019, no. 4, pp. 2-8. doi:10.31799/1684-8853-2019-4-2-8 